\title{
MODELO MULTICRITÉRIO DE DECISÃO PARA LOCALIZAÇÃO DE NOVA JAGUARIBARA COM VIP ANALYSIS
}

\author{
Vanessa Ribeiro Campos \\ Adiel Teixeira de Almeida * \\ Universidade Federal de Pernambuco \\ Recife - PE \\ nessarc@fortalnet.com.br \\ aalmeida@ufpe.br \\ * Corresponding author / autor para quem as correspondências devem ser encaminhadas \\ Recebido em 12/2004; aceito em 10/2005 \\ Received December 2004; accepted October 2005
}

\section{Resumo}

Projetos de grandes barragens, na maioria das vezes, implicam inundação de cidades e povoados pelo lago formado. A complexidade do processo de decisão de localizar a população atingida pela barragem decorre de uma interação de variados critérios, em geral, conflitantes, bem como da grande quantidade de atores envolvidos no processo. Este artigo considera um modelo de auxílio à tomada de decisão para o estudo de localização da cidade de Nova Jaguaribara, no Estado Ceará, sendo abordados diferentes pontos de vista técnicos, socioeconômicos e ambientais.

Palavras-chave: projetos públicos; localização; apoio multicritério a decisão, VIP Analysis.

\begin{abstract}
Projects of great dams, most of the time, imply in the flood of cities and populated areas because of the lake that is formed by the dam. The complexity of this decision problem, the location analysis of the population removed from the area is related to the interaction of different conflicting criterias and the great amount of actors involved in the process. This paper considers a model of Multicriteria Decision Aid for the study location of the city of Nova Jaguaribara in Ceará State, that involve different points of view: technological; socioeconomic; environment.
\end{abstract}

Keywords: location; public projects; multicriteria decision aid; VIP Analysis. 


\section{Introdução}

Os projetos de grandes barragens, na maioria das vezes, implicam inundação de cidades ou povoados pelo lago formado. Assim, um dos principais e mais debatidos projetos complementares ao projeto da barragem está relacionado ao reassentamento das populações atingidas pela barragem. Na decisão a ser tomada, local para construção de uma cidade, estão envolvidos as entidades públicas, moradores do local atingido e diversos critérios técnicos, econômicos, ambientais e sociais.

Diante desta realidade, muitos problemas são enfrentados pelos decisores quando avaliam a localização de uma cidade, pois envolve uma interação de critérios diferentes, em geral, conflitantes, fazendo com que haja dificuldade na tomada de decisão. Além disso, qualquer tomada de decisão nessa ordem pode resultar em um grande descontentamento da população, podendo inclusive resultar em um fracasso do projeto.

Pode-se dizer que o problema de localização de um centro urbano é tipicamente multicritério, pois envolve uma série de aspectos culturais, ambientais, econômicos, sociais e técnicos que devem ser considerados. Além disso, ao se estruturar um problema desta natureza, podem-se incorporar ao modelo, as preferências dos atores. Também não se pode deixar de considerar as preferências da população, a principal impactada pela sua remoção de seu habitat.

\section{Descrição do Problema}

A construção da barragem Castanhão, localizada no vale do Rio Jaguaribe foi tema de muita discussão desde o início do século XX, como solução para amenizar o problema da seca no Estado do Ceará. Os primeiros estudos para o projeto Castanhão foram realizados em 1910 pelo IOCS - Inspetoria de Obras Contra as Secas, sendo atualmente o Departamento de Obras Contra as Secas. O Castanhão tinha dois objetivos principais: garantir água durante os períodos críticos de seca do Estado e conter as enchentes nos anos especialmente chuvosos.

Entre as medidas a serem executadas com as obras do complexo Castanhão estava a construção de uma cidade de Nova Jaguaribara. A providência da construção de um espaço urbano tornou-se necessária uma vez que o núcleo urbano de Jaguaribara e a comunidade de Poço Comprido seriam inundados pela bacia hidráulica do Açude.

O estudo de caso tem por finalidade abordar o processo de decisão da localização da cidade de Nova Jaguaribara. O processo de tomada de decisão para a localização da cidade nova e o reassentamento de várias famílias foi bastante complexo. Esta medida significava várias mudanças sob inúmeros aspectos a serem considerados, de natureza social, econômica e ambiental. Os habitantes dos núcleos urbanos situados à margem do rio Jaguaribe tinham um forte elo de ligação com o rio que era utilizado para fins econômicos e de atividades de lazer. Isto fez com que houvesse a necessidade de atuação de diversos profissionais como: engenheiros, sociólogos, assistentes sociais, urbanistas, arquitetos, botânicos, geógrafos, representantes da igreja.

A partir da análise dos aspectos fisiográficos da área, procedeu-se à escolha do sítio para implantação da cidade de Nova Jaguaribara. Para isto foram consideradas, neste processo, como principais elementos direcionadores, as condições ambientais, os aspectos 
socioeconômicos locacionais; buscando-se a alternativa que viabilizasse o nascimento de uma cidade ecologicamente equilibrada, socialmente justa e economicamente viável (Oliveira et al., 2001).

Conforme Roy (1996), a decisão sobre infra-estrutura pública é considerada irreversível e única. Estes tipos de decisões requerem grandes gastos e causam impactos significantes na vida dos indivíduos e no meio ambiente.

\section{Estruturação do Problema}

\subsection{Identificação dos atores}

Durante toda a relocação da população atingida pela obra, procurou-se informar e consultar sobre todas as medidas a serem adotadas com o objetivo de atender as reivindicações desta. A necessidade de envolvimento da população é fundamental, haja vista a intensidade de mudanças ocasionadas pela relocação dos moradores.

A participação da população apresenta-se em duas fases do modelo de decisão proposto. Em primeiro lugar, durante a exposição das alternativas de localização para a nova cidade. Esta exposição dever ser o mais transparente possível de modo que todos os moradores possam compreender quais as vantagens e desvantagens de cada alternativa.

A próxima fase configura a obtenção de informações sobre as preferências dos moradores da cidade de Jaguaribara e da vila de Poço Comprido. Existem várias maneiras de envolver a população no processo de decisório. Para isso, foi adotado o resultado do plebiscito no modelo proposto.

O Projeto Castanhão exigiu participação de diversos pontos de vistas no processo decisório já que os impactos causados pelo projeto eram altos e de natureza complexa. Diante de tais fatos, deve-se incorporar ao modelo todo um conhecimento técnico de fatores não percebidos pela população.

Os principais dados para análise foram obtidos de uma planta cartográfica da região e o Estudo para Relocação da Cidade de Jaguaribara (DNOCS, 1992). A partir destes dados, entrevistou-se diferentes especialistas para definição e análise dos critérios do modelo, entre eles: arquiteto, engenheiros especializados em recursos hídricos, geotecnia, saneamento e meio ambiente.

\subsection{Definição das alternativas potenciais}

Foram estudadas seis alternativas para a relocação da cidade nova de Jaguaribara definidas pelo DNOCS (1992). Neste relatório, foi realizado um estudo em cada alternativa considerando suas características físicas, como geologia, relevo dos solos, recursos, vegetação. Além disso, verificou-se o potencial econômico para cada alternativa, ou seja, as condições que cada local poderia oferecer para um crescimento econômico e social da população.

Estas alternativas definidas pelo DNOCS podem ser visualizadas pela Figura 1 e estão descritas na Tabela 1. 


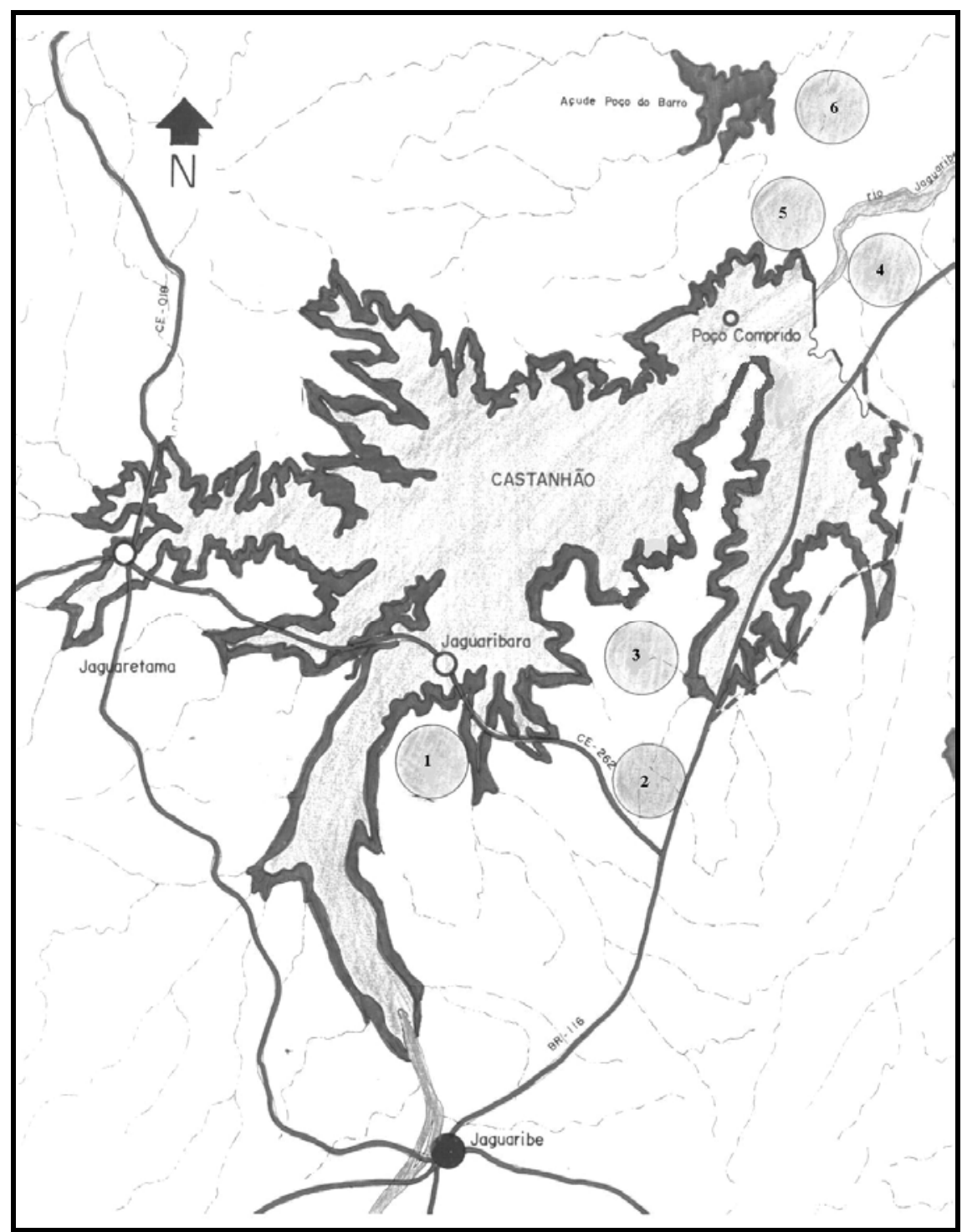

Figura 1 - Mapa de localização das alternativas.

\subsection{Critérios de avaliação}

Em muitos problemas de decisão, verifica-se que há mais de um objetivo a ser atingido. Este conjunto de objetivos chama-se conjunto de critérios ou atributos. Os critérios são utilizados como parâmetros de avaliação para o conjunto A de alternativas. Através da definição dos critérios do problema podem-se utilizar estes para fazer comparações entre as alternativas. Vincke (1992) define critério como uma função g, definida no conjunto A, que atribui valores de ordenação do conjunto A, e representa as preferências do decisor segundo o seu ponto de vista. 
Os Métodos de apoio multicritério a decisão tem como princípio buscar o estabelecimento de uma relação de preferências (subjetivas) entre as alternativas que estão sendo avaliadas sob a influência de vários critérios, no processo de decisão (Gomes, 2002; Almeida \& Costa, 2003).

Tabela 1 - Descrição das alternativas de localização de Nova Jaguaribara.

\begin{tabular}{cl}
\hline Alternativa & \multicolumn{1}{c}{ Localização } \\
\hline 1 & $\begin{array}{l}\text { Localizada a sudeste da margem direita do açude Público } \\
\text { Castanhão, no município de Jaguaribara. }\end{array}$ \\
\hline $\mathbf{2}$ & $\begin{array}{l}\text { Situada à margem direita da BR-116, encontra-se totalmente } \\
\text { inclusa no Município de Jaguaribara. }\end{array}$ \\
\hline $\mathbf{3}$ & $\begin{array}{l}\text { Localiza-se no Município de Jaguaribara, a sudeste da } \\
\text { península que aparecerá após a formação do açude Castanhão. }\end{array}$ \\
\hline $\mathbf{5}$ & $\begin{array}{l}\text { Localizada a jusante do barramento e à margem direita do rio } \\
\text { Jaguaribe. Encontra-se totalmente inserida no município de } \\
\text { Alto Santo. }\end{array}$ \\
\hline $\mathbf{6}$ & $\begin{array}{l}\text { Situada à jusante da barragem, mas na margem esquerda do } \\
\text { Rio Jaguaribe. Abrange terras no município de Alto Santo e } \\
\text { Morada Nova, sendo parte dessas terras pertencentes ao futuro } \\
\text { Projeto de Jaguaribara/ Castanhão. }\end{array}$ \\
\hline & $\begin{array}{l}\text { Localizada no município de Morada Nova, nas proximidades } \\
\text { do distrito de Poço do Barro e abrangendo uma porção da } \\
\text { mancha irrigável do Projeto Jaguaribara/ Castanhão. }\end{array}$ \\
\hline
\end{tabular}

Adaptado de DNOCS (1992)

Para o estudo deste problema foi desenvolvido o modelo apresentado na Figura 2, que considera os diferentes atores no processo decisório. Neste modelo pode-se agrupar os critérios em duas categorias: aqueles percebidos pela população e aqueles não percebidos pela população, que são avaliados pelos especialistas. A Figura 2 apresenta o fluxograma de definição dos critérios.

O fluxograma indica as características dos critérios de avaliação. Na avaliação da localização para a nova cidade. Os critérios não divididos em duas categorias: aqueles percebidos pela população que corresponde as suas preferências; aqueles não percebidos pela população ou que não são de interesse desta.

\subsubsection{Critério percebido e de interesse da população}

O primeiro critério de avaliação corresponde ao resultado do plebiscito realizado pelo DNOCS. Neste critério considerou-se o percentual de votos para cada alternativa de localização. Observa-se que este critério representa naturalmente uma escala cardinal (Gomes, 2002). 


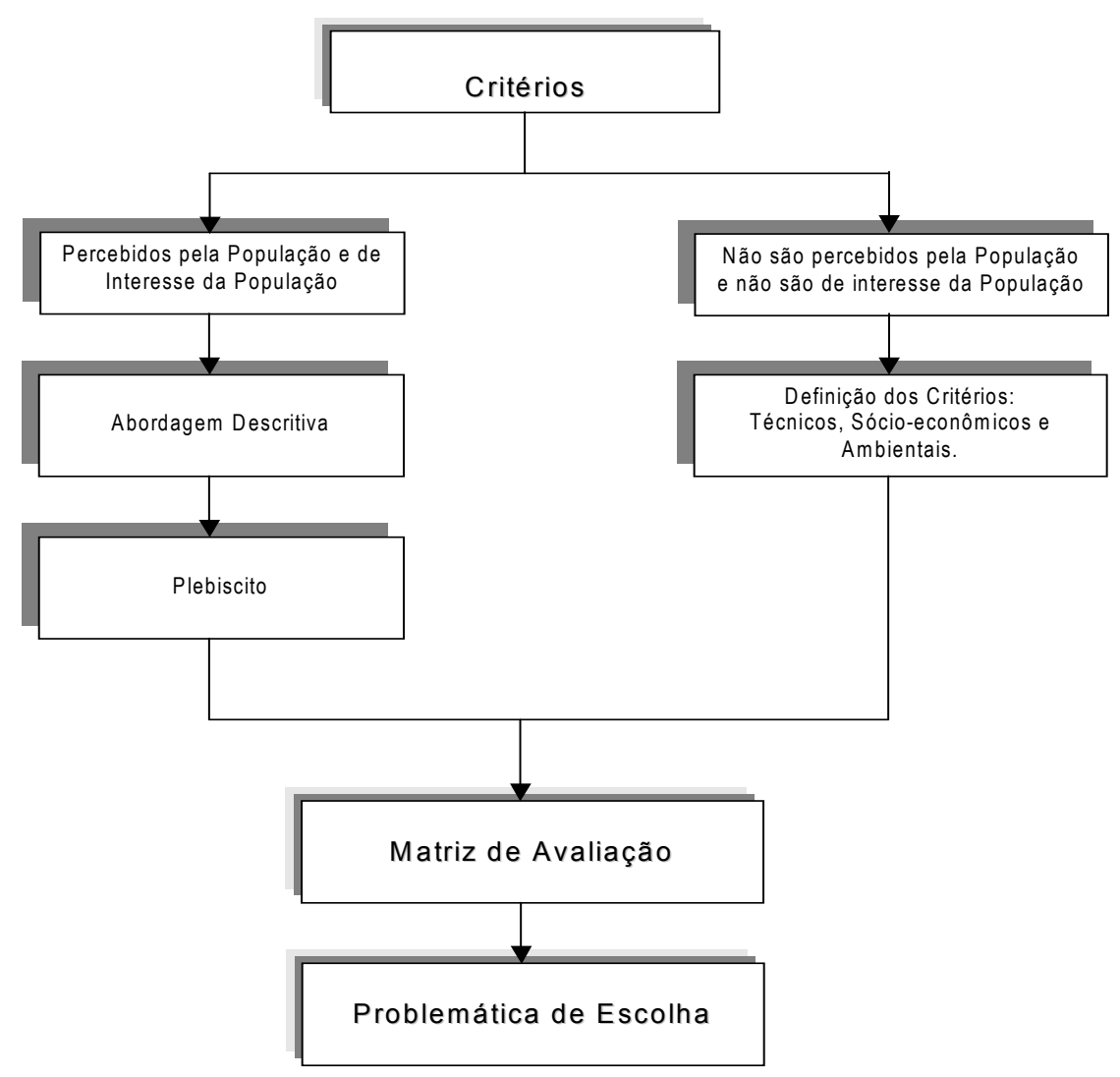

Figura 2 - Fluxograma de definição dos critérios de avaliação.

É importante ressaltar que esses dados fizeram parte da primeira sondagem de consulta a população.

Tabela 2 - Resultado do plebiscito.

\begin{tabular}{ccc}
\hline Alternativa & Número de Respostas & Percentual (\%) \\
\hline 1 & 13 & 2,6 \\
2 & 25 & 5,0 \\
3 & 01 & 0,2 \\
4 & 28 & 5,6 \\
5 & 329 & 65,8 \\
6 & 61 & 12,2 \\
Não responderam & 41 & 8,2 \\
Outras alternativas & 02 & 0,4 \\
Total & 500 & 100,0 \\
\hline & & Fonte: DNOCS (1992)
\end{tabular}




\subsubsection{Critérios não percebidos pela população}

Para cada critério a seguir é importante observar que foi obtida uma avaliação em escala cardinal. Este foi um dos requisitos necessários para a utilização do método aplicado. Nos casos onde se apresenta uma escala verbal, ou uma escala numérica de 1 a 5 (ou de 1 a 10), deve-se destacar que a diferença de atratividade entre níveis adjacentes é igual (Gomes, 2002).

\section{a) Aspectos técnicos}

A análise técnica tem por objetivo analisar as características existentes na região do ponto de vista de engenharia. A definição dos critérios técnicos envolveu especialistas em geotecnia e hidrologia para avaliar as condições de cada alternativa.

- Critério 2 - Tipo de solo considerando sua drenagem

O segundo critério a ser considerado foi o tipo de solo para construção de um centro urbano. Este critério tem por finalidade avaliar o quanto a drenagem do solo é propícia à construção de um núcleo urbano com construções de pequeno e médio porte cuja características são baseadas na antiga cidade. Considera-se que, quanto mais impermeável for o solo, mais propício para construção civil. O estabelecimento deste critério baseou-se em uma nota em uma escala de um a cinco, sendo cinco considerado aquele solo em melhores condições para construção, ou seja, aquele pouco drenado.

- Critério 3 - Tipo de solo considerando sua textura

Este critério também tem como finalidade avaliar o tipo de solo mais propício à construção civil, neste caso considera-se a textura do solo. Neste critério, utilizou uma escala de um a dez, pois foram vários os fatores levados em consideração (granulomeria, erosão, afloramento e outros). A nota máxima (10) foi atribuída para o solo com melhores condições para construção civil. Observa-se que para o espaço de alternativas analisado neste problema, não foram encontrados casos que se enquadrassem nos níveis mínimos e máximos desta escala.

- Critério 4 - Potencial hídrico

Neste caso foi considerado o ponto de vista hidrológico de cada alternativa para fins de obtenção de água na cidade. O potencial hídrico considerado foi tanto superficial (pequenos açudes, riachos) como subterrâneo. Aqui foi estabelecida uma nota de um a cinco, sendo o maior número aquele que maior potencial hídrico.

b) Aspectos socioeconômicos

O impacto social para a população relocada é de estimação difícil. Neste contexto, foram considerados apenas alguns critérios com a finalidade de abordar fatores sociais e econômicos que incidem sobre a população relocada.

- Critério 5 - Acessibilidade

A implantação de uma cidade requer a existência de uma boa infra-estrutura viária. Assim, este critério tem por finalidade avaliar as condições de infra-estrutura de estrada existentes na época de definição das alternativas. Este critério foi classificado como socioeconômico por questões comerciais, oportunidades de turismo, entre outras questões. Neste critério, utilizou uma escala verbal com os 5 níveis (desprezível, fraca, regular, boa, muito boa). 
- Critério 6 - Potencial econômico

Tem a finalidade de avaliar o potencial econômico da população, em outras palavras, avaliar as oportunidades de emprego para cada localidade. Entre as possibilidades de geração de empregos estão: pesca, turismo, comércio com municípios vizinhos e agricultura. A fim de avaliar as alternativas de localização foi estabelecida uma nota de um a dez, sendo a nota maior dada para aquela alternativa em que apresentasse maiores oportunidades de emprego. Observa-se que para o espaço de alternativas analisado neste problema, não foram encontrados casos que se enquadrassem no nível máximos desta escala.

\section{c) Aspectos ambientais}

A construção de um centro urbano requer todo um estudo de impacto ambiental. $\mathrm{Na}$ construção de um centro urbano, a alteração no meio ambiente é inevitável, mas deve-se procurar amenizar este impacto ao máximo possível.

\section{- Critério 7: Desmatamento}

Diante dos dados disponíveis no problema, ao analisar a localização das alternativas é fundamental considerar o desmatamento, que será ocasionado pela construção do empreendimento. A alteração do meio incidirá diretamente no meio biótico e abiótico da região. Neste contexto, foi considerada a infra-estrutura na época e foi avaliado o grau de desmatamento para a construção de uma cidade em cada localidade. Neste critério foi avaliado o grau de desmatamento por meio de uma escala verbal, considerando 4 níveis: altíssimo, alto, médio e baixo.

- Critério 8: Despejo de efluentes tratados

Quando se faz um planejamento urbano deve-se considerar o local de despejo dos efluentes tratados. O local ideal para o despejo dos efluentes dever ser a jusante do rio Jaguaribe para poderem ser depurados. Os efluentes tratados não podem ser jogados a montante do açude, pois podem poluir a bacia hidrográfica formada pela barragem. Levando em consideração o posicionamento das alternativas, foi atribuída uma nota de um a cinco para o local mais propício para o despejo destes efluentes, sendo a maior dada para aquela opção que era mais favorável ao despejo de efluentes tratados, portanto, causará menor impacto no meio ambiente.

\subsection{Matriz de avaliação}

Também chamada matriz de decisão, é aquela que apresenta as alternativas com relação aos $n$ critérios de avaliação, auxiliando à tomada de decisão.

Tabela 3 - Matriz de avaliação.

\begin{tabular}{|c|c|c|c|c|c|c|c|c|}
\hline Critérios & $\begin{array}{c}\text { População } \\
1\end{array}$ & \multicolumn{7}{|c|}{ Técnicos } \\
\hline Alternativa & $\begin{array}{c}\text { Plebiscito (\% } \\
\text { de votos) }\end{array}$ & Drenagem & Textura & $\begin{array}{c}\text { Potencial } \\
\text { Hídrico }\end{array}$ & $\begin{array}{c}\text { Potencial } \\
\text { Econômico }\end{array}$ & Acessibilidade & $\begin{array}{c}\text { Desmatame } \\
\text { nto }\end{array}$ & $\begin{array}{c}\text { Efluentes } \\
\text { tratados }\end{array}$ \\
\hline $\mathbf{1}$ & 2,6 & 2 & 3 & 2 & 4 & regular & médio & 2 \\
\hline $\mathbf{2}$ & 5 & 5 & 4 & 1 & 7 & muito boa & baixo & 3 \\
\hline $\mathbf{3}$ & 0,2 & 3 & 7 & 1 & 2 & desprezível & alto & 1 \\
\hline $\mathbf{4}$ & 5,6 & 4 & 4 & 3 & 6 & boa & baixo & 5 \\
\hline $\mathbf{5}$ & 65,8 & 1 & 5 & 5 & 8 & desprezível & altíssimo & 5 \\
\hline $\mathbf{6}$ & 12,1 & 4 & 8 & 4 & 1 & fraca & alto & 4 \\
\hline
\end{tabular}




\subsection{Caracterização da problemática}

A definição do tipo de problemática é uma etapa relevante na estruturação do problema, pois, pela definição da problemática pode-se compreender melhor o tipo de problema em questão. Roy (1996) define quatro tipos de problemáticas: escolha, descritiva, classificação e ordenação.

Neste estudo de caso, podem ser considerados dois tipos de problemática: num primeiro momento a problemática descritiva e num segundo, é de escolha (Figura 2). A problemática descritiva $(\mathrm{P} \delta)$ tem por finalidade descrever todas as alternativas do problema de modo que estas sejam apresentadas para todos os atores do problema. Por meio da abordagem de descrição, deve-se utilizar uma linguagem apropriada, de acordo com o nível de instrução dos moradores, para que eles compreendam todas as características das alternativas e as conseqüências que a escolha de cada localidade pode trazer.

Na próxima fase do modelo, tem-se a problemática de escolha cuja finalidade é selecionar reduzir o conjunto de alternativas, para este caso, apenas uma alternativa.

\subsection{Escolha do método multicritério}

O processo de escolha do método de multicritério a ser utilizado como apoio a decisão no problema depende de vários fatores tais como: as características do problema, o contexto, a estrutura de preferência do decisor, e o tipo de problemática (Almeida \& Costa, 2003).

A consideração de todos estes aspectos levou a escolha do método VIP Analysis. Especialmente em relação ao estudo do contexto, houve a influência da questão das informações imprecisas encontradas neste problema, pois neste tipo de problema e contexto é difícil atribuir pesos aos critérios de avaliação na escolha de localização da cidade de Nova Jaguaribara.

Apoio multicritério à decisão se baseia em método matemático que requer a escolha de um algoritmo. Esses modelos e o desempenho do algoritmo caracterizam-se por apresentar número $\mathrm{n}$ de parâmetros de natureza, muitas vezes, subjetivas. Atribuir valores a esses parâmetros é uma tarefa difícil já que esses valores muitas vezes estão sujeitos a incertezas e imprecisões (Dias \& Clímaco, 2000b).

O método multicritério de agregação aditiva VIP Analysis, torna-se bastante eficiente para o tipo de problema abordado, considerando informações parciais. De acordo com Dias \& Clímaco (2000a), este método permite auxiliar os tomadores de decisão encontrar as alternativas preferidas. A construção dessa função requer que seja fixada uma ordem de importância dos critérios, ou seja, uma ordenação para as constantes de escala. O método permite extrair conclusões a respeito das alternativas sem a necessidade de atribuição de pesos ou constantes de escadas.

Outra questão fundamental foi a constatação de independência preferencial entre os critérios neste problema, o que permite o uso de um modelo de agregação aditivo (Vincke, 1992; Gomes, 2002; Almeida \& Costa, 2003). Condição necessária para o uso do método VIP Analysis.

Outro aspecto observado, em relação à estrutura de preferências do decisor foi a possibilidade de efetuar compensação entre os diferentes critérios o que possibilitava o uso de uma função utilidade aditiva, somada ao atendimento à condição de independência preferencial. Ou seja, a estrutura de preferências dos atores envolvidos atende a uma lógica compensatória (Vincke, 1992; Gomes, 2002; Almeida \& Costa, 2003). Esta condição elimina 
a possibilidade de utilização do método ELECTRE IV, que seria apropriado numa situação de considerando informações parciais, mas requer uma visão não-compensatória.

Outra vantagem do método selecionado está relacionada à facilidade de uso e compreensão pelos atores do processo, além da disponibilidade da ferramenta computacional de apoio ao método.

\section{Método de Agregação Aditiva com VIP Analysis}

O modelo multicritério de agregação aditiva VIP Analysis (Variable Interdependent Parameters) tem como finalidade trabalhar com informações imprecisas (Dias \& Clímaco, 2000a) e é apresentado a seguir.

Uma função aditiva de agregação como múltiplos critérios pode ser representada pela expressão (1).

$$
\mathrm{V}\left(\mathrm{a}_{\mathrm{i}}, \mathrm{k}\right)=\sum_{\mathrm{j}=1}^{\mathrm{n}} \mathrm{k}_{\mathrm{j}} \mathrm{u}_{\mathrm{j}}\left(\mathrm{g}_{\mathrm{ij}}\right)
$$

Sejam:

$A$ Conjunto de alternativas; sejam $\mathrm{m}$ alternativas $\mathrm{a}_{1}, \mathrm{a}_{2}, \ldots, \mathrm{a}_{\mathrm{m}}$.

C Conjunto de pontos de vistas considerados, ou seja, denominados de critérios ou atributos; sejam n critérios.

$\mathrm{g}_{\mathrm{ij}}$ Performance de i-ésimo elemento do conjunto A de acordo com o j-ésimo critério ou atributo.

$\mathrm{u}_{\mathrm{j}}($.$) Função valor com único atributo associada ao \mathrm{j}$-ésimo atributo assinala um valor numérico a cada performance, tal que $\mathrm{u}_{\mathrm{j}}\left(\mathrm{g}_{\mathrm{xj}}\right)>\mathrm{u}_{\mathrm{j}}\left(\mathrm{g}_{\mathrm{yj}}\right)$ se a performance de $\mathrm{g}_{\mathrm{xj}}$ é melhor que a performance de $g_{\mathrm{yj}}, \mathrm{e} \mathrm{u}_{\mathrm{j}}\left(\mathrm{g}_{\mathrm{xj}}\right)=\mathrm{uj}\left(\mathrm{g}_{\mathrm{yj}}\right)$ se as performances de ambas são equivalentes.

$k_{\mathrm{j}} \quad$ Importância do parâmetro (constante de escala) associada à $\mathrm{u}_{\mathrm{j}}($.$) .$

$K$ Conjunto de combinações aceitáveis de valores para as constantes de escalas em um determinado estádio do processo de apoio a decisão (cada combinação é representada por um vetor de $k \in K$ ). Considera-se aqui que os elementos desse conjunto devem concordar com as restrições: $k_{\mathrm{j}} \geq 0$ e $\sum_{j=1}^{n} k_{j}=1$.

Sendo o elemento $\mathrm{k}=\left(k_{1}, k_{2}, \ldots, k_{n}\right)$ pertence ao conjunto $K$ e corresponde a informação imprecisa relativa a importância dos parâmetros.

Sejam duas alternativas $\mathrm{a}_{\mathrm{x}}$ e $\mathrm{a}_{\mathrm{y}}$ do conjunto $A$ e Ot $\left(\mathrm{a}_{\mathrm{x}}\right)$ se refere ao subconjunto de $\mathrm{K}$ em que o valor de $\mathrm{a}_{\mathrm{x}}$ é maior que (ou igual a) o valor das outras alternativas do conjunto $A$. Pode-se, então, apresentar os seguintes conceitos:

- dominância absoluta $-\mathrm{a}_{\mathrm{x}} \Delta_{A} \mathrm{a}_{\mathrm{y}} \Leftrightarrow V\left(\mathrm{a}_{\mathrm{x}}, k\right) \geq V\left(\mathrm{a}_{\mathrm{y}}, k\right) \forall k \in K$ e $\exists k, k^{J} \in K$ : $V\left(\mathrm{a}_{\mathrm{x}}, k\right)>V\left(\mathrm{a}_{\mathrm{y}}, k\right)$

- dominância (Bernoulli) - $\mathrm{a}_{\mathrm{x}} \Delta \mathrm{a}_{\mathrm{y}} \Leftrightarrow V\left(\mathrm{a}_{\mathrm{x}}, k\right) \geq V\left(\mathrm{a}_{\mathrm{y}}, k\right) \forall k \in K$ e $\exists k \in K$ : $V\left(\mathrm{a}_{\mathrm{x}}, k\right)>V\left(\mathrm{a}_{\mathrm{y}}, k\right)$

- otimização de $k \in K-\mathrm{a}_{\mathrm{x}}$ é ótima em $k \Leftrightarrow V\left(\mathrm{a}_{\mathrm{x}}, k\right) \geq V\left(\mathrm{a}_{\mathrm{y}}, k\right) \forall \mathrm{a}_{\mathrm{y}} \in A \backslash\left\{\mathrm{a}_{\mathrm{x}}\right\} \Leftrightarrow$ $k \in \operatorname{Ot}\left(\mathrm{a}_{\mathrm{x}}\right)$;

- potencialmente ótima (p.o.) $-\mathrm{a}_{\mathrm{x}}$ é p. o. $\Leftrightarrow \exists k \in K: \mathrm{a}_{\mathrm{y}} \in A \backslash\left\{\mathrm{a}_{\mathrm{x}}\right\}, V\left(\mathrm{a}_{\mathrm{x}}, k\right) \geq$ $V\left(\mathrm{a}_{\mathrm{y}}, k\right) \Leftrightarrow \mathrm{Ot}\left(\mathrm{a}_{\mathrm{x}}\right)$. 
O programa VIP Analysis (Dias \& Clímaco, 2000a), pode ser encontrado no endereço: http://www4.fe.uc.pt/lmcdias/software.htm.

Dias \& Clímaco (2005) acentuam que esta ferramenta tem como objetivo apoiar a avaliação de um conjunto de alternativas de forma a escolher a mais preferida, de acordo com a função aditiva, conforme segue, sendo $v_{\mathrm{j}}\left(\mathrm{a}_{\mathrm{i}}\right)$, o valor de $\mathrm{a}_{\mathrm{i}}$ para o critério $\mathrm{j}$.

$$
\begin{aligned}
& \mathrm{V}\left(\mathrm{a}_{\mathrm{i}}, \mathrm{k}\right)=\sum_{\mathrm{j}=1}^{\mathrm{n}} \mathrm{k}_{\mathrm{j}} \mathrm{v}_{\mathrm{j}}\left(\mathrm{a}_{\mathrm{i}}\right), \\
& \text { para } \sum_{j=1}^{n} k_{j}=1 \text { e } k_{\mathrm{j}} \geq 0 .
\end{aligned}
$$

O VIP Analysis permite calcular o intervalo de valor global de cada alternativa e a matriz de confrontação extrema, em que as alternativas são confrontadas aos pares. Nesta matriz, podem ser identificadas as alternativas dominadas ou "quasi-dominadas", assim como o valor máximo regret associado à escolha de cada alternativa e os domínios onde cada uma delas, sendo ótima ou "quasi-ótima" (caso o problema permita). As informações imprecisas sobre critérios são tratadas por intermédio de um conjunto de restrições a que os valores dos coeficientes de escala devem obedecer.

\section{Aplicação do VIP Analysis no Estudo de Localização de Nova Jaguaribara}

A etapa inicial de aplicação do método de agregação aditiva VIP Analysis inclui a transformação de escala da matriz de avaliação (Tabela 3). Ao se utilizar o programa VIP Analysis, pode-se transformar todos os critérios em uma escala numérica de modo que se tenham valores de zero a um, o que pode ser feito para as escalas cardinais utilizadas. Esta informação é apresentada na Figura 3.

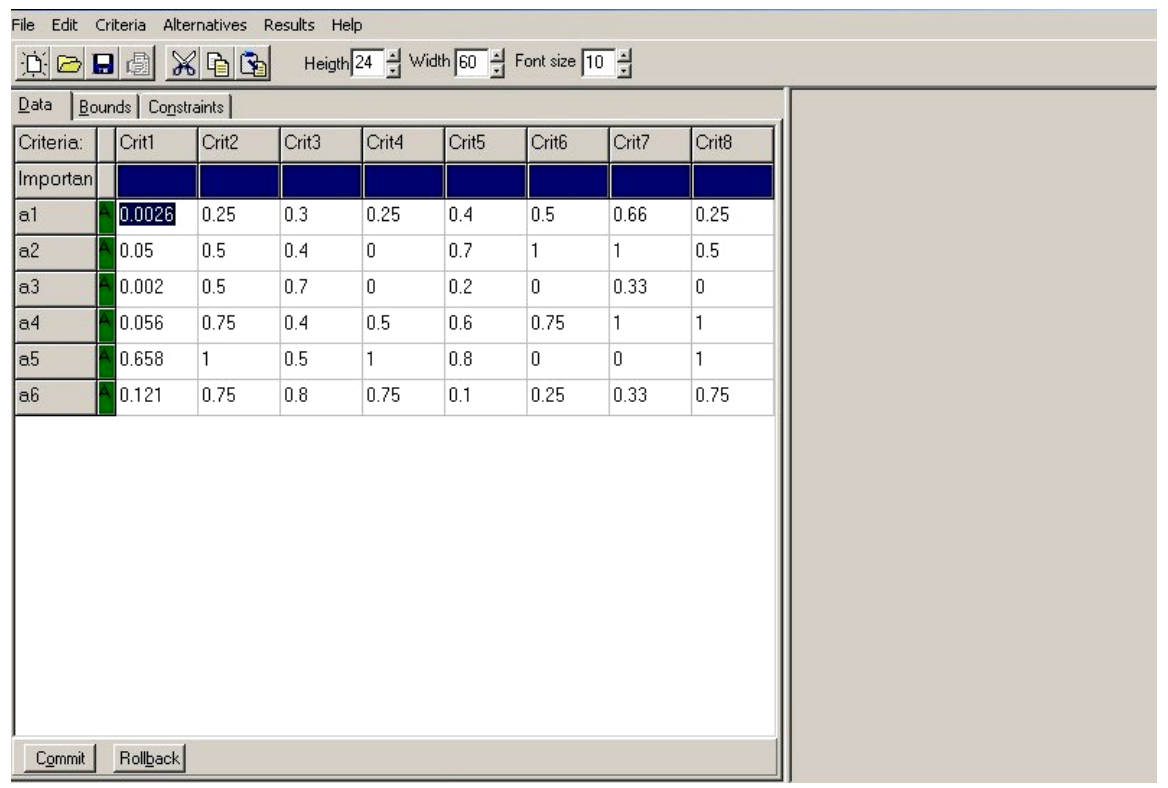

Figura 3 - Matriz de avaliação normalizada no VIP Analysis. 
Em seguida, analisam-se as informações do problema que estão relacionadas com os "pesos dos critérios". Para isso, foi consultado o decisor e verificada a ordem das constantes de escala $(k)$. O decisor tratou-se de alguém que participou diretamente nas ações e tomadas de decisão para a construção da nova cidade, com experiência, tanto na definição e análises das alternativas, como no envolvimento com a comunidade.

É importante observar que foi considerada a identificação dos níveis de desempenho de referência (ou âncoras) para estabelecimento da avaliação das constantes de escala, mesmo que, sob condição de informação parcial. Esta questão é importante para métodos compensatórios, em função do próprio significado das constantes de escala (parâmetros associados ao grau de importância dos critérios), conforme já abordado na literatura (Keeney \& Raiffa, 1976; Keeney, 1992; Gomes, 2002; Almeida \& Costa, 2003). Foi visto, com o agente decisor, que a avaliação da constante de escala para cada critério, deveria considerar os valores reais de desempenho em cada critério representados pelos valores limites da escala $(0,1)$ na Figura 3. Assim, o trade-off, representado pelas avaliações relativas das constantes de escala, representa o grau de troca em cada critério com base nos valores de desempenho real em cada critério, para este problema.

Observou-se ainda que, os valores de constantes de escala não representam diretamente a importância dos critérios, como ocorre em métodos não-compensatórios (Vincke, 1992; Gomes, 2002; Almeida \& Costa, 2003). Nos métodos compensatórios, como é o caso do modelo aditivo no VIP Analysis, os valores de constantes de escala estão associados à importância dos critérios e à faixa de valores de desempenho do critério para o espaço de alternativas na escala (no caso de 0 a 1) de avaliação (Keeney \& Raiffa, 1976; Keeney, 1992). Assim, para facilidade de linguagem e na análise dos resultados a seguir, quando se menciona que um critério é mais importante do que outro e sua constante de escala (ou peso) é maior, tem-se em mente que isto está condicionado ao contexto de faixa de valores de desempenho real dos critérios para a escala de 0 a 1 usada.

De acordo com as entrevistas com o decisor ficou estabelecida a seguinte ordem para as constantes de escala:

$$
\left.\left.\left.\left.\left.\left.\left.\mathrm{k}_{6}\right\rangle \mathrm{k}_{5}\right\rangle \mathrm{k}_{1}\right\rangle \mathrm{k}_{2}\right\rangle \mathrm{k}_{3}\right\rangle \mathrm{k}_{4}\right\rangle \mathrm{k}_{8}\right\rangle \mathrm{k}_{7}
$$

Observa-se pela ordem de critérios estabelecida que o decisor valorizou inicialmente os critérios sócio-econômicos, em seguida, técnicos e, por último, ambientais. O critério mais importante para o decisor foi à acessibilidade, ou seja, a existência de infra-estrutura local da região. A razão dada pelo decisor foi a necessidade de aproveitamento da infra-estrutura existente no local. Constata-se também, na expressão 3, que os critérios ambientais ( $k_{8}$ e $\left.k_{7}\right)$ ocupam posições finais na ordem de importância definida pelo decisor. O critério $k_{l}$, representando as preferências da população, ocupa uma posição de preferência após os critérios de acessibilidade e potencial econômico. Esta ordem de importância para os critérios será inserida no VIP Analysis influenciando, diretamente, a escolha de localização para a nova cidade.

A ordem das constantes de escala são inseridas no VIP Analysis por meio de uma série de restrições, sendo $k_{1}+k_{2}+k_{3}+k_{4}+k_{5}+k_{6}+k_{7}+k_{8}=1$. As sete restrições que provêm desta ordenação: $k_{5}-k_{6} \leq 0,\left(-k_{5}\right)+k_{1} \leq 0,\left(-k_{1}\right)+k_{2} \leq 0,\left(-k_{2}\right)+k_{3} \leq 0,\left(-k_{3}\right)+k_{4} \leq 0,\left(-k_{4}\right)+k_{8} \leq 0$, $\left(-k_{8}\right)+k_{7} \leq 0$. Essas sete restrições definem a ordem de preferência estabelecida pelo decisor ora apresentado,ou seja, $\mathrm{k}_{6} \geq \mathrm{k}_{5} \geq \mathrm{k}_{1} \geq \mathrm{k}_{2} \geq \mathrm{k}_{3} \geq \mathrm{k}_{4} \geq \mathrm{k}_{8} \geq \mathrm{k}_{7}$. 


\subsection{Resultados do VIP Analysis na visão do decisor}

Definida essa ordem, obtém-se todas as informações necessárias para utilização do programa VIP Analysis. A primeira abordagem refere-se à amplitude de cada alternativa, ou seja, o mínimo e o máximo valor global de que cada alternativa pode apresentar.

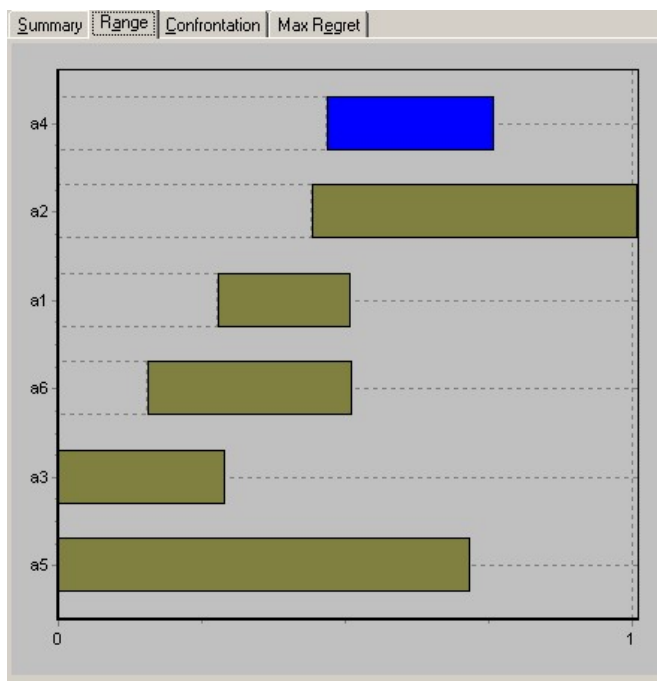

Figura 3 - Amplitude de valor global das alternativas.

A Figura 3 mostra uma ordenação das alternativas segundo o mínimo valor global. Nesta figura pode-se visualizar que a alternativa superior (a4) é aquela que apresenta o maior valor mínimo global. Pode-se constatar, também, que a3 apresenta um máximo valor global bem inferior ao mínimo valor global de a4, sendo, portanto, considerada absolutamente dominada. A Figura 4 mostra que as alternativas 1, 3 e 6 são dominadas, pois seus valores máximos globais estão próximos ao o valor mínimo de a4. Além disso, averigua-se, pelo resumo, que a4, além de apresentar um valor mínimo global superior às demais, possui um máximo regret inferior a todas alternativas.

\begin{tabular}{|c|c|c|c|c|c|}
\hline Summary & Range & Confrontation & Max Regret | & & \\
\hline Alternative & Value & Min Value & Max Value & Max Regret & Dominated? \\
\hline a1 & & 0.279 & 0.5 & 0.5 & YES \\
\hline$a 2$ & & 0.442 & 1 & 0.258 & \\
\hline 23 & & 0 & 0.28 & 1 & YES (Abs) \\
\hline a4 & & 0.469 & 0.75 & 0.25 & \\
\hline$a 5$ & & 0 & 0.708 & 1 & \\
\hline 96 & & 0.157 & 0.503 & 0.75 & YES \\
\hline
\end{tabular}

Figura 4 - Resumo dos resultados do VIP Analysis na visão do decisor.

O programa VIP Analysis disponibiliza uma tabela com a comparação par a par entre as alternativas, chamada de matriz de confrontação. Nas células desta matriz encontra-se máxima vantagem que uma alternativa pode apresentar em relação à outra, ou seja, a diferença de valor entre pares de alternativas (Figura 5). 


\begin{tabular}{l|l|l|l|l|l|l|}
\hline & $\mathrm{a} 1$ & $\mathrm{a} 2$ & $\mathrm{a} 3$ & $\mathrm{a} 4$ & $\mathrm{a} 5$ & $\mathrm{a} 6$ \\
\hline $\mathrm{a} 1$ & & -0.158 & 0.5 & -0.168 & 0.5 & 0.275 \\
\hline $\mathrm{a} 2$ & 0.5 & & 1 & 0.25 & 1 & 0.75 \\
\hline $\mathrm{a} 3$ & -0.01 & -0.208 & & -0.231 & 0 & -0.075 \\
\hline $\mathrm{a} 4$ & 0.305 & 0.129 & 0.75 & & 0.75 & 0.5 \\
\hline $\mathrm{a} 5$ & 0.429 & 0.258 & 0.508 & 0.15 & & 0.329 \\
\hline $\mathrm{a} 6$ & 0.224 & 0.053 & 0.303 & -0.047 & 0.25 & \\
\hline Max Regret: & 0.5 & 0.258 & 1 & 0.25 & 1 & 0.75 \\
\hline
\end{tabular}

Figura 5 - Matriz de confrontação.

As células que apresentam números na cor vermelha denotam que a alternativa indicada pela linha é dominada pelas alternativas mostradas pela coluna. Observa-se na quarta coluna a existência de três células vermelhas, isto demonstra que a4 domina as alternativas 1, 3 e 6 . Verifica-se, então, que a opção 4 apresenta maior quantidade de alternativas dominadas por ela. Portanto, nesta análise, considerando as preferências do decisor, a alternativa 4 tem um melhor desempenho que as demais.

Outro fato importante a ser destacado está relacionado com a quinta coluna, a5 não domina, no entanto, não é dominada por qualquer alternativa. Porém, o seu valor máximo de arrependimento é muito alto.

O máximo regret corresponde ao máximo arrependimento para cada alternativa caso esta seja escolhida pode ser visto na Figura 4. Observa-se pelo gráfico acima que a2 e a4 apresentam valores muito próximos de máximo regret.

\subsection{Análise de diferentes cenários}

A análise de localização de Nova Jaguaribara pode ser ampliada com a definição de novos cenários de decisão. Estes cenários compreendem situações em que o ponto de vista do decisor em relação à ordem de importância das constantes de escala difere da situação inicial. Esta análise tem como objetivo avaliar a performance das alternativas para diferentes situações.

\section{a) Cenário 1}

Como foi observado o decisor considerou o maior grau de importância os critérios socioeconômicos, em seguida, técnicos e, por último, ambientais. No primeiro cenário, esta situação foi invertida, os critérios mais importantes são aqueles de caráter ambiental, depois, socioeconômicos e, finalmente, os técnicos. A expressão 4 indica a ordem de preferência para as constantes de escala.

$$
k_{8}>k_{7}>k_{6}>k_{5}>k_{1}>k_{2}>k_{3}>k_{4}
$$

Vale ressaltar que esta ordem preferência foi alterada apenas de acordo com as características dos critérios (ambientais, socioeconômicos e técnicos). Pela expressão 4, o decisor estabeleceu uma ordem para os critérios ambientais $k_{7}$ e $k_{8}$ que não foi alterada, ou seja, $k_{7}$ é mais importante que $k_{8}$, sendo esta ordem respeitada para ambos os cenários.

O resumo indicado pela Figura 6 mostra que as alternativas 1, 2, 3 e 6 são dominadas pela alternativa 4. Nota-se, também, que, nesta nova situação, a4 apresenta-se novamente em destaque. Deste modo, a4 tem melhor desempenho, pois seu valor mínimo global é superior 
as demais alternativas. A alternativa 5, apesar de não estar dominada apresenta um valor mínimo global bem inferior à a4 .

\begin{tabular}{|c|c|c|c|c|}
\hline \multicolumn{5}{|c|}{ Ślmmary |Range | Confrontation | Max Regret | } \\
\hline Alternative & \begin{tabular}{|l|l|l} 
Value Min Value \\
\end{tabular} & Maxvalue & Max Regret & Dominated? \\
\hline a1 & 0.25 & 0.47 & 0.75 & YES (Abs) \\
\hline $\mathrm{a} 2$ & 0.5 & 0.833 & 0.5 & YES \\
\hline a3 & 0 & 0.247 & 1 & YES (Abs) \\
\hline $\mathrm{a4}$ & 0.632 & 1 & 0 & \\
\hline a5 & 0.333 & 1 & 0.583 & \\
\hline$a 6$ & 0.31 & 0.75 & 0.48 & YES \\
\hline
\end{tabular}

Figura 6 - Resumo do VIP Analysis para o cenário 1.

\section{b) Cenário 2}

Neste cenário foram atribuídos como critérios mais importantes os fatores técnicos de localização, em seqüência, fatores socioeconômicos e, finalmente, fatores ambientais. $\mathrm{O}$ segundo cenário é definido pela expressão 5 :

$$
k_{2}>k_{3}>k_{4}>k_{6}>k_{5}>k_{1}>k_{8}>k_{7}
$$

A Figura 7 cenário indica que a5 apresenta mínimo valor global superior às demais alternativas. Nota-se uma diferença entre os valores mínimos globais de a5 e a4 é muito pequena mostrando, portanto, um bom desempenho de a4. Neste caso, a1, a2 e a3 são absolutamente dominadas, pois possuem valores máximos globais inferiores ao valor mínimo global de a5. Observa-se pela Figura 7 que a ordenação das alternativas é bem diferente das situações anteriores, cuja alternativa em destaque é a5.

\begin{tabular}{|c|c|c|c|c|c|}
\hline \multicolumn{6}{|c|}{ Summary |Rạnge | Confrontation | Max Regret | } \\
\hline Alternative & Value & Min Value & Maxvalue & Max Regret & Dominated? \\
\hline a1 & & 0.25 & 0.34 & 0.75 & YES (Abs) \\
\hline a2 & & 0.3 & 0.52 & 0.533 & YES (Abs) \\
\hline a3 & & 0.2 & 0.6 & 0.508 & YES (Abs) \\
\hline a4 & & 0.509 & 0.75 & 0.283 & \\
\hline a5 & & 0.62 & 1 & 0.025 & \\
\hline$a 6$ & & 0.462 & 0.775 & 0.25 & \\
\hline
\end{tabular}

Figura 7 - Resumo do VIP Analysis para o cenário 2.

Os resultados do VIP Analysis para este cenário indicam que a5 tem uma "melhor" performance quando os critérios de avaliação técnicos são mais importantes na tomada de decisão. Além disso, pode-se constatar que a4 (alternativa escolhida nas situações anteriores) também apresenta uma boa performance neste cenário. 


\section{Resultados da Aplicação do VIP Analysis}

Pelos resultados obtidos podem-se tirar as seguintes conclusões:

- as alternativas 1,3 e 6 são dominadas segundo a ordenação das constantes de escala definida pelo decisor;

- na primeira situação, a2 e a4 apresentam valores mínimos globais semelhantes, no entanto, a4 destaca-se por em todos os resultados do VIP Analysis como a matriz de confrontação e o máximo regret;

- a4 apresenta um bom desempenho para diferentes situações em que a ordenação das constantes de escala é alterada. Esse fato é confirmado nas três situações analisadas, em que, apesar da alteração na ordenação, a4 apresentou boa performance em todas elas;

- a5, apesar de não ser dominada em nenhuma situação, é uma alternativa que apresenta variações de na sua performance de acordo com a preferências do decisor;

- o programa VIP Analysis mostrou-se bastante eficiente no auxilio à tomada decisão com informações imprecisas.

De acordo com essa análise, pode-se concluir que a alternativa 4 é a mais indicada para a localização de Nova Jaguaribara. Assim, a localização mais indicada para Nova Jaguaribara, segundo as preferências do decisor é a jusante da barragem do açude Castanhão, situada à margem esquerda do Rio Jaguaribe.

Vale ressaltar que atualmente a cidade de Nova Jaguaribara se encontra localizada no local proposto pela alternativa 5 . Esta decisão baseou-se apenas nas preferências da população.

\section{Conclusões}

O deslocamento involuntário de uma população para dar lugar a construções de infraestrutura pública é um problema de decisão de natureza bastante complexa. A remoção da população pode ocasionar grandes insatisfações que comprometem o sucesso do projeto. Além disso, um projeto dessa dimensão requer a interação de vários especialistas de áreas diferentes.

Deve-se ser incluído no processo decisório de projetos públicos vários critérios necessários o estudo eficiente da localização em que irá abrigar a população impactada. A utilização de um método multicritério de apoio à decisão trouxe significativas vantagens para a abordagem do problema, pois é possível incorporar ao modelo uma variedade de pontos de vistas, inclusive as preferências da população.

Neste artigo foi proposto um modelo multicritério de decisão para resolução do problema de localização da cidade de Nova Jaguaribara. Na definição do modelo, foram evidenciados os principais atores do processo de decisão, os critérios de avaliação do problema, o tipo de problemática. Essas definições são fundamentais para a estruturação e entendimento do problema focalizado.

A aplicação do método multicritério de decisão agregação aditiva VIP Analysis mostrou-se bastante eficiente ao estudo de informações imprecisas, foi possível evoluir e tirar conclusões a respeito das alternativas sem a necessidade de atribuição de pesos aos critérios. $\mathrm{O}$ método multicritério permitiu auxiliar a tomada de decisão no sentido de resolver o conflito entre os critérios de avaliação. 
O estudo de comportamento das alternativas diante de outros cenários estabelecidos para ordenação das constantes de escala foi de grande valia. A partir deste estudo foi possível verificar a performance das alternativas diante de perfis diferentes de decisor.

Verificou-se que no cenário em que critérios ambientais eram mais importantes, os resultados da aplicação do VIP Analysis indicaram novamente a escolha da alternativa 4. No segundo cenário estabelecido, em que os critérios técnicos seriam os mais valorizados, os resultados do programa apontaram a alternativa 5 como melhor, no entanto, a performance da alternativa 4 foi semelhante a esta alternativa. $\mathrm{O}$ estabelecimento destes cenários serviu para confirmar $\mathrm{o}$ desempenho da alternativa 4 diante das demais alternativas. Conclui-se, portanto, que o local situado à jusante do barramento e à margem direita do rio Jaguaribe foi o mais indicado para a construção da cidade de Nova Jaguaribara segundo as preferências do decisor.

\section{Agradecimentos}

Este trabalho foi parcialmente apoiado pelo CNPq e pela CAPES e teve expressivo apoio e contribuição do Eng. Francisco Pardailan Farias da SRH e da Arq. Raquel Cristina Pontes, na realização deste estudo.

\section{Referências Bibliográficas}

(1) Almeida, A.T. \& Costa, A.P.C.S. (2003). Aplicações com Métodos Multicritério de Apoio a Decisão. Universitária UFPE, Recife.

(2) Dias, L.C. \& Clímaco, J.N. (2000a). Additive Aggregation with Variable Interdependent Parameters: the VIP Analysis Software. Journal of Operational Research Society, 51(9), 1070-1082.

(3) . (2000b). Shortest Path Problems with Partial Information: Models and Algorithms for Detecting Dominance. European Journal of Operational Research, 121, 16-31.

(4) (2005). Dealing with Imprecise Information in Group Multicriteria Decisions: a Methodology and a GDSS Architecture. European Journal of Operational Research, 160, 291-307.

(5) DNOCS (1992). Estudo de Relocação da Cidade de Jaguaribara. Relatório.

(6) Gomes, L.F.A.M.; Gomes, C.F.S. \& Almeida, A.T. (2002). Tomada de Decisão Gerencial: Enfoque Multicritério. Editora Atlas, Rio de Janeiro.

(7) Keeney, R. \& Raiffa, H. (1976). Decision with Multiple Objectives: Preferences and Value Trade-offs. John Wiley \& Sons.

(8) Keeney, R.L. (1992). Value Focused Thinking. Havard.

(9) Oliveira, M.C. \& Cabral L.X. (2001). Nova Jaguaribara: Uma Medida Mitiadora de Impactos Sociais e Humanos do "Castanhão". XXIV Seminário Nacional de Grandes Barragens.

(10) SRH (1999). Contribuição da Barragem do Castanhão para o Desenvolvimento Sustentável do Ceará. Relatório Técnico, Secretaria dos Recursos Hídricos do Estado do Ceará.

(11) Vincke, P. (1992). Multicriteria Decision-aid. John Wiley \& Sons. 